Voix et Images

voixetimages

\title{
La littérature québécoise à Voix et Images : créneau ou ghetto?
}

\section{Bernard Andrès}

Volume 12, numéro 2 (35), hiver 1987

Jacques Brault

URI : https://id.erudit.org/iderudit/200639ar

DOI : https://doi.org/10.7202/200639ar

Aller au sommaire du numéro

\section{Éditeur(s)}

Université du Québec à Montréal

\section{ISSN}

0318-9201 (imprimé)

1705-933X (numérique)

Découvrir la revue

\section{Citer cet article}

Andrès, B. (1987). La littérature québécoise à Voix et Images : créneau ou ghetto? Voix et Images, 12(2), 303-312. https://doi.org/10.7202/200639ar d'utilisation que vous pouvez consulter en ligne.

https://apropos.erudit.org/fr/usagers/politique-dutilisation/ 


\title{
La littérature québécoise à Voix et images: créneau ou ghetto?
}

\author{
par Bernard Andrès, Universitẻ du Québec à Montréal
}

Créneau: Segment de marchés ou gamme de besoins auxquels un type de produit ou de service convient particulièrement (cf. Larousse).

Ghet to: Endroit sù une minorité est séparée du reste de la société; condition déclassée ou marginate d'un milieu refermé sur luimême (cf. Larousse).

Littérature québécoise; Placée dans la connexité de la littérature d'expression fransaise dans la France d'outre-mer et à l'étranger (cf. Histoire des littératures dans la Pléiade).

Voix et images: La seule revue universitaire du Quéhec exclusivement consacrée au domaine littéraire et culturel quéhécois (cf. la publicité de la revue).

Après le témoignage critique de Jacques Allard sur les origines de Voix et images (de 1967 à 1975), j'aborderai la période des onze dernières années, en m'interrogeant sur le statut et le traitement de notre corpus en termes de "créneau» et de méthode. Que représente la littérature québécoise dans les trente-cinq derniers numéros: comment l'a-t-on cernée (au double sens de l'approche et de la limite, de la théorie et du territoire)? 
Du repli sur le terroir, au structuralisme et aux applications de grilles, jusqu'à l'ouverture comparatiste, comment s'est effectué le cheminement critique dans cette revue qui frôle aujourd'hui (si l'on remonte aux "Cahiers du Sainte-Marie "), vingt ans d'existence? Fondées sur l'examen des sommaires, des (rares) avant-propos et de certains articles de fond, mes observations ne constituent qu'un bilan préliminaire. Seule l'analyse de l'Index raisonné de Voix et images, qui paraîtra prochainement, permettra d'affiner, de nuancer et d'approfondir cette modeste présentation de 6000 pages d'études, de chroniques et de notes bibliographiques auxquelles contribuèrent pas moins de 350 chercheur-e-s'.

Je ne m'attarderai pas à l'aspect économique de la revue. Quelles que soient nos prétentions à verser dans la recherche désintéressée, nous ne pouvons comme revue universitaire négliger le marché, les abonné-e-s, les ventes en kiosque et notre image auprès des lecteurs; avec un handicap de taille: rester sérieux, respectable, austère presque, alors que nos collègues des magazines jouent dans la polychromie, le tabloïd, l'icone et le graphisme débridé. Les produits Spirale, Vice versa, Arcade etc. sont, dans ce sens, plus comestibles. Même Liberté s'amuse avec sa maquette... Mais sommes-nous vraiment concurrents? Ils font leur travail, nous faisons le nôtre, dans un contexte économique de plus en plus critique. Car la récession'a frappé. C'est l'heure des «vaches maigres" du côté des subventionneurs qui considèrent comme un luxe la coexistence de trois revues universitaires de littérature au Québec. Pourtant les revues résistent. Elles font front commun et tablent sur leur compétence et leur complémentarité. Voix et images a son créneau, elle doit s'y tenir. Il y a vingt ans. l'équipe fondatrice ne pensait pas en termes de marketing ou de créneau: c'est moins par contrainte de marché que par choix, culturel ou idéologique, que les fondateurs et les collaborateurs de la première heure créèrent la collection Voix et images du pays dans les "Cahiers de Sainte-Marie». C'est dans le même esprit que la collection se constituait en revue dès 1975 et qu'André Vanasse relançait en 1981 la publication en introduisant des numéros entièrement thématiques au besoin.

Rappelons certains jalons de cette politique éditoriale, Quand Jacques Allard ouvre le premier volume de Voix et images (nouvelle formule), en septembre 1975, il intitule son avant-propos "Pour la continuité":

Une nouvelle revue? Les fidèles lecteurs de Voix et images du pays auront vite fait de s'y reconnaître. Derrière la nouveauté du format (plus économique), ils retrouveront vite l'essentiel de la collection qu'ils apprécient depuis 1967: l'objectif fondamental n'a pas changé qui consiste à publier des études consacrées exclusivement à la production québécoise.

La nouveauté se marque surtout en ceci: l'entrevue avec un écrivain (artiste, cinéaste ou autre producteur) reviendra dorénavant à chaque livraison; de même notre rec'ension des publications utiles aux chercheurs du domaine québécois, et nos chroniques sur les dernières parutions, le cinéma ou les autres domaines de 
l'activité culturelle. Ainsi, la revuue ne fera que plus ș̣stématiquement ce que réalisait déjà la collection d'études.

Ce qui frappe, malgré tout. c'est la détermination avec laquelle on ampute le titre de la revue du troisième terme de l'ancienne triade ("du pays"): $L a$ thématique du pal's des années soixante a vécu. Même s'il est encore question de "discours national", l'accent est mis sur le texte québécois, sur des questions de méthodologie, sur des modes d'approches du fait littéraire (de "l'histoire littéraire " à "l'analyse textuelle ", en passant par "l'analyse idéologique" et "les sciences humaines").

La création est bien mentionnée au passage, mais sans le même enthousiasme éditorial que sous l'ancienne formule. Il est vrai que les revues de création québécoise sont déjà bien établies et qu'il n'est pas question de faire double usage 2 . On ne relèvera donc que quelques inédits, au hasard des trente-quatre (34) numéros suivants de Voix et images ${ }^{3}$. Par contre, l'analyse textuelle prend vite le dessus sur les études thématiques. C'est ce que révèle un examen sommaire de la titrologie d'articles des années 1975-1980. Faute d'espace, je me contenterai de mentionner l'importance relative des catégories d'approches indexées par les titres les plus récurrents ${ }^{4}$.

Les articles les plus marqués au coin de la thématique sont produits dans les années 1975 à 1979. Parallèlement aux problématiques identitaires sur le pays (décidément toujours vivaces):

- La recherche du pays chez $X$...

- X et l'identité québécoise...

- Vers la découverte de lidentité... (1977)

- L'idéologie du pays dans... (1979),

on lit des études thématiques sur «la concupiscence et l'avarice», «l'espace et le délire", "le précieux et la préciosité», "la femme", «la ville", «l'eau», etc. Pendant cette période, cependant, l'inspiration des autres articles est majoritairement structurale, voire techniciste. Ils portent sur des corpus restreints, dans une perspective surtout synchronique. Tel texte est un sistème à décoder, et l'on ne compte plus les énoncés du type:

"Déchiffrons l'Antiphonaire", "Lecture structurale de...". "Thèmes et structures de...", "Étude de la structure anaphorique dans...", "Forme et signification du $X$ dans...", etc.

Mais déjà la sémiologie a pris la relève: études du discours, analyses rhétoriques, bientôt relayées par la pragmatique et les questions d'énonciation, vont bon train jusqu’à la fin des années 1970:

"Le Texte comme dire», "Clôture du moi, clôture du texte", "Les 26 figures d'une rhétorique de soi", "Rhétorique de l'histoire: essai de modèle ", "Interprétation de quelques formes de discours dans...», "Sémiologie de l'ironie», "la Question de l'énonciation en...", "le Signe 'silence' dans...", "Sémiologie et poétique" "Essai d’analyse sémiotique de...", jusqu’à cette superbe "Sémiologie d'un ange, étude...n, etc. 
Aux confins de la sémiotique, la narratologie et l'analyse actantielle font : bon ménage avec l'approche sociocritique, ce jusqu'à 1984 environ:

"Théorie actantielle et processus idéologique ...", "Voix auctoriale et voix narrative dans...", "Masques narratifs et contre-masques idéologiques", "l'Auteur implicite», "Dịstance et point de vue, voix et idéologie dans...", etc.

Les années 1980 voient fleurir l'analyse' institutionnelle et les premiers bilans sur les rapports entretenus entre la littérature et le social. André Belleau publie en 1983 son fameux article sur «la Démarche sociocritique au Québec». en dénonçant certains abus sociologisants de la méthode:

Notons d'abord l'existence au Québec d'une asociocritique " traditionnelle, une sorte de discours sociologisant général pratiqué depuis roujours par les journalistes, les écrivains, les professeurs, qui tend à considérer chaque ceuvre importante parue comme un moment réussi ou non d'une révolution inséparable de l'évolution nationale et politique. (...) on a souvent tendance à se rabattre sur le social quand il y' a pauvreté ou pénurie de signifiant. ${ }^{5}$

D’autres travaux, moins nombreux, mais présents jusqu'au début des années 1980, s'inscrivent dans une perspective bachelardienne. mauronienne, mythocritique ou psychanalytique du phénomène littéraire. C'est l'article de Gérard Bessette dans le tout premier numéro de 1975 ( «la Psychocritique»), mais aussi :

"L'Êcriture comme pratique fantasmatique», "la Politique du désir chez...", "Circularité de la rêverie chez...", "Littérature populaire religieuse: esquisse socio-psychanalytique", "X et l'enveloppe des mythes", "X ou l'histoire au service du mythe", etc,

ainsi que la savoureuse étude en deux volets de Henri-Paul Jacques:

«Le cas C. Le Cassé sur le tchesteurfilde, un cas pour les psychanalystes [dit-il] ou d'une esthétique du bâton, S-T-Tick: stick [dit-il ]» (!)

À la croisée de l'une ou de l'autre de ces approches, une dizaine d'études abordent de façon diachronique des corpus plus vastes, des mouvements ou des questions d'histoire et de genres littéraires:

"X et la fiction au XIX $\mathrm{X}^{\mathrm{p}}$, "Typologie du discours pamphlétaire", "l'Histoire des idées au Québec", "le Récit court de type exemplum », "les Romanciers du XIX ${ }^{\mathrm{e}}$ siècle face à..." " "la Censure au Québec", "l'Essai au Québec», "les Romans historiques de...", "le Titre du roman québécois dans les années....).

C'est à partir de 1977 que les études d'inspiration féministe se manifestent de façon régulière à Voix et images. Au nombre d'un article par an en moyenne, jusqu’à 1981', ce type de recherche se développe et se diversifie 
nettement à partir de 1982. Parties là àussi de la thématique, de la sociologie ou de l'histoire littéraire:

«la Femme et le nationalisme dans...», «la Condition féminine dans...", «les Écrivaines dans l'histoire littéraire québécoișe», "Théâtre au féminin", " Mise à mort de la femme et libération de l'homme", "le Reflet mère-fille dans...", etc.

ces recherches se nourrissent aussi bien de l'analyse du discours que des études comparées:

"l'Intertextualité dans les écrits d'inspiration féministe», «Margaret Atwood et Nicole Brossard", ou le récent article d'Élène Cliche sur Clarice Lispector, Madeleine Gagnon et France Théoret (no 34).

Ce qui nous amène à l'orientation la plus récente repérable dans notre revue: les études comparées. Bien que spécialisée en littérature québécoise, Voix et images a toujours cultivé d'excellents rapports avec les chercheurs du reste du Canada, comme en témoigne dès le premier volume la liste des collaborateurs et des correspondants. En 1977, un correspondant des ÉtatsUnis se joignait à l'équipe (Jonathan M. Weiss), suivi en 1980 d'une Italienne (Franca Marcato), en 1984 d'un Français et d'une Brésilienne (Guy Lecomte et Lilian Pestre de Almeida) et, dernièrement, d'une Mexicaine et d'un Polonais (Laura Lopez Morales et Jerzy Parvi). Cette représentation du milieu international n'est que le reflet de notre lectorat puisque les dernières statistiques indiquent une certaine hausse des abonnements à l'étranger, par rapport au rythme de croissance des abonnements québécois. Néanmoins cette ouverture du monde aux lettres québécoises n'était pas payée en retour d'un intérêt de nos chercheurs pour les aires culturelles voisines, canadiennes ou américaines (au sens large). Il y avait bien eu dans Voix et images du pays, en 1975, l'«Esquisse d'une sémantique du créole haütien et dú joual français", de Maximilien Laroches, mais il faut attendre 1983 pour trouver dans Voix et images "le Mythe des ethnies dans les romans de l'ouest canadien (...)", de E.D. Blodget. Nous sommes sous le mandat d'André Vanasse. L'article est suivi en 1984 de "l'Image du roman québécois en France», de Jacqueline Barrois Gérols $(1 X, 2)$ et du premier dossier consacré à "la Littérature canadienne-anglaise" (X, I).

L'impulsion était donnée. Elle s'accentue sous le mandat de votre serviteur avec coup sur coup, en 1986, «la Réception de la littérature canadiennefrançaise en France au XIX ${ }^{\mathrm{e}}$ siècle», de Pierre Hébert et surtout le premier dossier comparatiste du tout récent no 34. Dans la livraison de l'automne passé, six chercheurs-e-s du Québec et d'Amérique du Sud s'interrogent sur la dimension latino-américaine de productions brésiliennes, argentines, mais aussi québécoises: le discours littéraire américain et son rapport au code européen, les notions de tiers-mondisme, de marronnage culturel et d'hybridisation, d'intertexte transculturel, ou du régime de représentation de la réalité et des discours dans des productions romanesques issues d'aires cultu- 
relles différentes. Ringuet, Hubert Aquin, Madeleine Gagnon, France Théoret, Jacques Godbout se retrouvent sous d'autres latitudes, en d'autres compagnies: Graciliano Ramos, Julio Cortázar, Clarice Lispector, Moacyr Scliar... Et Gilles Thérien (lecteur de Borges) de revendiquer pour le Québec la position du tiers, de prendre conscience de la totalité de l'Amérique, Nouveau Monde d'un pôle à l'autre, terre de conquête et de métissage où l'Europe est venue organiser horizontalement un territoire vertical ${ }^{9}$.

On voit le chemin parcouru depuis 1967, époque où la collection d'études se présentait ainsi:

Pour être impérative, l'entreprise n'en demeure pas moins immense, qui parviendra peut-être un jour à saisir dans sa totalité le plain-chant et l'imagerie du pa!s réalisé ou conquis.

Vingt ans après, en dépit des aléas du nationalisme ou, qui sait?, grâce au désenchantement de l'après-référendum, une nouvelle vision du pays, plus continentale, mieux ouverte à l'hybridisation des discours et des cultures, est peut-être en train de se forger. Elle coïncide avec un phénomène d'institutionnalisation de l'appareil critique. Désormais, chaque département y va de son projet en littérature québécoise. Les centres d'études québécoises, les regroupements de chercheurs s'installent. se développent. entrent en concurrence, essaiment à l'étranger: Bruxelles, Paris, Rio, Varsovie, Bangkok, sans compter les États-Unis où les lettres québécoises deviennent la seconde spécialisation "in" des jeunes professeurs en mal de carrière. The American Council for Quebec Studies a sa propre revue dans le New Hampshire: Québec Studies. À quand la bulle papale qui partagera le petit monde des "Quebec Scholars around the World"? Ici, au Québec, les études québécoises ne cessent de se consolider. En ce qui nous concerne, les liens de Voix et images avec les centres d'études à l'étranger témoignent de notre conception du fameux segment de marché. Pour nous, créneau n'est pas ghetto.

Les conditions socio-professionnelles et institutionnelles de la recherche en lettres québécoises sont un des autres points intéressants à examiner. Le profil type du chercheur a passablement changé en vingt ans. Quand la production québécoise s'est accrue et diversifiée (boom de l'édition, impact des subventions, etc.), la recherche universitaire s'est affinée. Elle a dû se spécialiser, non plus seulement vingtième siècle versus dix-neuvième siècle, mais les années 1960, l'entre-deux guerres, les années 1970 , tel mouvement, tel auteur et, depuis peu, le rapatriement et la redécouverte des textes de Nouvelle France. De nombreux chercheurs se sont réorientés, réalignés sur le Québec. Quand les directeurs et animateurs actuels de nos revues (qu'elles soient ou non spécialisées en québécois) ont "gradué» dans les années 60-70, leurs thèses portaient sur Camus, Proust, Zola, Claude Simon, Valéry, etc. Ils se sont dirigés ensuite ou parallèlement, selon les cas, vers le corpus québécois : Bessette, Hébert, Beaulieu, Godbout, Ferron prennent alors la place de Valéry, Zola et consorts. On se tourne aujourd'hui vers Brossard, Tremblay, Villemaire et la nouvelle écriture. Subséquemment pourtant, l'université s'est refermée. Elle n’embauche plus ou presque. Les diplômé-e-s en lettres québé- 
coises, sauf exception, se retrouvent chargé-e-s de cours ou professeure-e-s de cegep. Bien souvent ils n'analysent plus la littérature sur laquelle on les a formés, ils la font ou, plutôt. ils en font une autre, dite nouvelle, postmoderne. Ils la publient dans leur maison à eux, qu'ils commentent entre eux dans leurs propres revues. leurs propres magazines, s'étonnant presque d'être couverts à l'occasion dans nos revues institutionnelles. Le petit débat sur notre numéro " $\mathrm{BJ} / \mathrm{NBJ}$ ", en 1985 , témoigne bien du malaise qui étreint le milieu lorsque deux circuits de légitimation se court-circuitent. Le problème est crucial à l'heure actuelle. Un clivage de générations sous-tendu par des données socio-professionnelles nouvelles, écarte du champ littéraire, du champ de la recherche surtout. une masse de jeunes intellectuels que nous avons pourtant formés. Le milieu n'est plus aussi homogène que jadis: les clivages se multiplient et. signe des temps, à l'intérieur d'une même génération. Les instances critiques se multiplient également et, avec la nouvelle écriture, les références théoriques suivent le pas. Sans prendre à la lettre toute prétention à la modernité (car on patauge souvent dans les rebuts des autres avantgardes), il faut constater un certain nombre de jalons dans le parcours théorique québécois depuis vingt ans.

Chaque corpus génère son approche. On l’a vu, lâge de la parole a connu. la thématique, socio et psycho-critique ont suivi de près, d'abord fortement teintées de thématisme, même quand il était question de débusquer "l'image-Québec dans la poésie de $X$ " ou "la quête d'identité dans le personnage de $Y^{\prime}$. L' histoire de ces filiations théoriques (de certaines fixations théoriques, épistémologiques également) reste à faire. À quand la thèse sur la fortune de Goldmann au Québec? Sur le passage du concept de vision du monde à celui d'idéologème? de production du signifiant ou de déconstruction discursive? Un phénomène étonne toutefois lorsque l'on fouille dans les références théoriques convoquées par les articles de nos revues et leurs fameuses notes infra-paginales: la prédominance de la tradition européenne (française surtout ou anglaise, allemande, italienne mais en traduction française). Peu ou pas de références étrangères dans le texte ou encore de références américaines, au sens large. C'est peut-être là que réside le danger de créneau, vécu comme ghetto par les chercheurs aussi bien en littérature québécoise que française. Je ne suis pas loin de penser que le danger n'est pas dans la prédilection pour un corpus précis, québécois ou non, car toute recherche sérieuse circonscrit nécessairement son objet, mais plutôt dans les limites méthodologiques qu'on s'impose en se fixant une aire géo-théorique sans considérations pour d'autres sphères et perspectives de réflexion. Quand cette aire de fixation est l'Europe, que cela confine à la France en particulier. le danger se double d'un syndrome de néo-colonialisme assez navrant.

Pourtant, le continent américain est tout proche: mieux, nous y sommes. Un bon nombre de chercheurs ne semblent pas encore le soupçonner. Les barrières linguistiques y sont pour quelque chose certes, mais aussi cette fâcheuse tendance à.regarder vers l'est plutôt que vers le sud. L'avenir n'est-il pas au comparatisme trans-américain? Avec des précautions méthodologiques bien sûr; une concordance de dates d'édition, un croisement thématique ne 
justifient aucun raccourci. «L'américanité» comme la "québécitude" peuvent être des mythes. Si l'américanité U.S. nous gêne (hantise de l'assimilation), pourquoi ne pas épouser la perspective latino-américaine? Hantise du tiersmondisme? Toutes ces littératures dont certaines sont à la pointe de ce qui se fait (en roman par exemple) n'ont-elles pas connu des problèmes analogues de colonisation, de post-colonisation, de rapports aux modes et aux codes européens? C'est là tout un programme que nous commençons à peine à entrevoir. Les problèmes d'épistémologie comparatiste doivent être posés dans une perspective québécoise. Tout reste à faire pour éviter l'effet de ghetto. Il ne s'agit pas d'élargir notre créneau vers la littérature française, par exemple, mais de creuser le créneau que nous occupons déjà, d'approfondir nos travaux, de mieux comprendre notre corpus en l'ouvrant au reste du continent. Avant de se lancer dans cette voie, il faut assurer nos arrières en maintenant coûte que coûte la formule de la revue. Elle a fait preuve depuis plus de dix ans: dossiers, études, chroniques. Je terminerai donc par un survol de nos principales rubriques.

Dans ses "Chroniques". Voix et images souligne les productions les plus marquantes de l'année, s'interroge au besoin sur leur discours d'accompagnement: c'est une actualité décantée qu'elle livre aux lecteurs. Dans ses "Études", la revue accentue cette distance par rapport à la primeur, à l'actualité. On couvre aussi bien un récit de voyage du dix-septième siècle qu'une œuvre contemporaine. Pour le texte contemporain. l'étude prend alors pour objet non seulement le texte littéraire en soi mais aussi les textes critiques qu'il a provoqués. L'étude fait le point sous un angle nouveau en soulevant des problèmes qui excèdent souvent les limites ou la portée du texte littéraire considéré. Les questions de méthodologie prennent le pas (narratologie, sociocritique, textanalyse, etc.). d'où l'aspect ardu de certains articles et parfois le danger d'écraser le texte sous la grille. Le risque est énorme dans le cas de certaines productions moins solides qui résistent mal aux machines de guerre «narratologeuses». J'ai moi-même usé un peu trop rudement du carré sémiotique à propos de la Terre paternelle de Lacombe ou de l'analyse actantielle dans Pour la patrie de Tardivel. On en est arrivé d'ailleurs à parler d'auteurs ou d'ceuvres prédestinées à la critique, d'une sorte de "littérature pour l'analyse", d'exemplum offert en pâturage au scalpel universitaire: comme Ricardou pour le nouveau roman, certains Bessette pour la psychocritique, tel Major pour la sociocritique, tel Aquin pour la sémiotique; les meilleures ouvres demeurant celles qui résistent le mieux à ces assauts.

Parallèlement à ces "Études" et à ces "Chroniques», Voix et images aborde également les auteurs dans des "Dossiers" sous forme d'entretien, d'articles et de bibliographies. C'est là sans doute que la revue rejoint le mieux sa fonction d'instance de légitimation, en mettant en vedette tel ou tel agent littéraire plus ou moins coté dans le milieu. Le choix de ces vedettes n’a pas toujours fait l'unanimité: trop de "vieux", ou, à l'inverse, de trop "jeunes" auteurs ou encore trop de professeurs, trop d'hommes, etc. Qu'en est-il vraiment? ${ }^{10}$ Sur trente-cing livraisons en onze ans et abstraction faite des deux numéros thématiques, on trouve vingt-cinq hommes pour sept femmes, quatorze auteurs reconnus (déjà primés) sur trente-deux dossiers, 
donc un bon équilibre (sauf pour ce qui est de la représentation féminine). Quant à la "jeunesse», rappelons que les entrevues et les dossiers consacrés à des auteurs "montants» sont assez nombreux (à l'époque où ces dossiers étaient créés): Chamberland. Brossard. Duguay. Beaulieu. Louis-Philippe Hébert, Haeck, Villemaire, etc. Quant aux professeurs d'université, ils ne totalisent que huit dossiers sur les trente et un (deux d'entre eux seulement émergeant de l'UQAM: deux poètes, Madeleine Gagnon et Michel van Schendel). Bon an mal an, Voix et images a donc occupé le créneau québécois, elle s'y est taillée un fief que viennent grignoter à l'occasion ses consœurs Études françaises et Etudes littéraires. Ce n'est pas inquiétant dans le sens où il ne s'agit pas pour nous d'un monopole, encore moins d'un ghetto, mais du champ d'étude prioritaire que nous comptons bien conserver et étendre, aux confins et au contact des autres littératures d'Amérique.

1. Pour des raisons évidentes de commodité et de longueur, je n’ai pu mentionner le nom de tous les auteur-e-s d'articles cités. Qu'ils soient, qu'elles soient ici remercié-e-s de leurs précieuses contributions à Voix et images. Je signale par ailleurs que j'abrègerai les références à notre revue par la seule mention du volume en chiffres romains, suivi du numéro en chiffres arabes, avec la mention de la saison et de l'année, ceci jusqu'au 2c numéro du volume XI qui devient à partir de 1986. le no 32. Exemple: XI. I. automne 1985: no 32. hiver 1986; no 33. printemps 1986. etc.

2. A titre indicatif et pour ne citer que ces deux revues. en 1975, la Barre du jour a dix ans d'existence et les Herbes rouges publient depuis sept ans déjà.

3. "L_cs Porches", poème inédit de Raoul Duguay (1. 2. décembre 1975);

Poèmes inédits de Guy Lafond (IV. 2, décembre 1978):

"Manuscrits trouvés dans une valise", de Louis-Philippe Hébert (IV. 3. avril 1979):

"Seize distances pour le doigt, l'ouvert. la page". de Michel van Schendel (no 32. hiver 1986):

"Yvel Swanson". de Yolande Villemaire (no 33, printemps 1986).

"Uma adore a Shiva". d'Elsa Cross (no 34. automne 1986).

A noter ćgalement deux timides tentatives d'insertion d'“essais" (confidences, témoignages, documents, etc.), intitulés Apartés: "Au ban des lettres, hors-texte et robeprétexte", de Gabrielle Poulin (V. 2. hiver 1980) et «la Meule et la rivière. souvenirs de Saint-Eustache". d"André Brochu (V. 3. printemps 1980). On aura remarqué que le principe de l'inédit réapparaît à partir du no 32 (1986).

4. Aux fins de cette présentation, je me permets de tronquer certains titres en ne conservant que les lexèmes et tours syntagmatiques les plus "parlants". en omettant les références à des corpus précis (remplacés par " $X$ "). Le relevé suivant ne porte que sur la section principale des "Études». Il va de soi que mes remarques devraient être complétées par une étude parallèle des sections "Dossier" et "Chroniques".

5. André Belleau, "la Démarche sociocritique au Québec". VIIl, 2. hiver 1983, p. 299-300.

6. Henri-Paul Jacques, in IV, I et 2, septembre et décembre 1978.

7. Voix et images publie annuellement une vingtaine d'études en moyenne.

8. Maximilien Laroche, in Voix et images du pays, IX, février 1975.

9. Gilles Thérien, in Voix et images, no 34, p. 14.

10. On consultera sur ce point la liste des auteurs abordés dans nos dossiers:

Hubert Aquin (I, 1); Raoul Duguay (I, 2); Gérard Bessette (I, 3); Fernand Leduc (II. 1); Paul Chamberland (II. 2); Jean Éthier-Blais (II, 3); Nicole Brossard (III, 1); Victor-Lévy Beaulieu (III, 2); Pierre Perrault (11I. 3); Rina Lasnier (IV, 1); Guy Lafond (IV, 2); Louis-Philippe Hébert (IV, 3); Jacques Godbout (V, 1); Yves Thé- 
riault (V. 2); Fernand Ouellette (V, 3); Gilles Marcotte (VI, I); Jean-Claude Germain (VI. 2); Philippe Haeck (VI, 3); Adrien Thério (VII. 1); Michel Tremblay (VII, 2); Anne Hébert (VII, 3); Madeleine Gagnon (VIII, I); Marie-Claire Blais (VIII, 2); Jacques Ferron (VIII, 3); Guy Dufresne (IX, 1); Roland Giguère (IX. 2); Monique Bosco (IX. 3); La littérature canadienne-anglaise (X. 1); La BJ/NBJ (X. 2); André Major (X. 3); Naïm Kattan (XI, 1); Michel van Schendel (32); Yolande Villemaire (33); Québec/Amérique latine (34) Jacques Brault (35) 